\title{
Magnetic Field Effect on Yb-Based Heavy Fermions near Magnetic-Nonmagnetic Transition
}

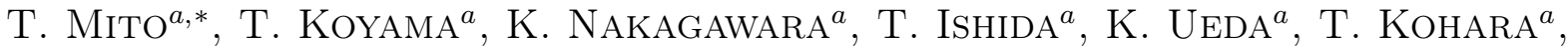

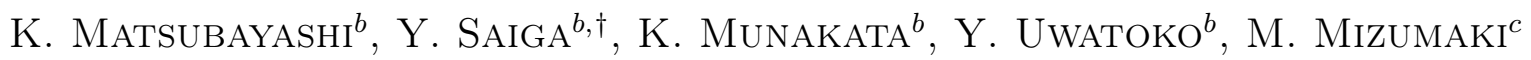 \\ AND N. KAWAMURA ${ }^{c}$ \\ ${ }^{a}$ Graduate School of Material Science, University of Hyogo, Hyogo 678-1297, Japan \\ ${ }^{b}$ Institute for Solid State Physics, University of Tokyo, Kashiwa, Chiba 277-8581, Japan \\ ${ }^{c}$ Japan Synchrotron Radiation Research Institute, SPring-8, Sayo, Hyogo 679-5198, Japan
}

\begin{abstract}
Recently magnetic field effects on $f$ electron systems in the vicinity of a magnetic-nonmagnetic transition have attracted much interest. In order to investigate the mechanism of field induced modifications in heavy fermion systems, especially in terms of valent degrees of freedom, we have carried out the X-ray absorption spectroscopy measurement on the newly discovered heavy fermion material $\mathrm{YbCo}_{2} \mathrm{Zn}_{20}$ which exhibits extremely large specific heat at low temperatures. Our results show that the $\mathrm{Yb}$ valence of $\mathrm{YbCo}_{2} \mathrm{Zn}_{20}$ is very close to +3 over the whole temperature range of measurement. Besides, no significant change in the absorption spectrum was observed up to the field of $10 \mathrm{~T}$. This contrasts to the previously reported strong field dependence in the resistivity, followed by an expansion of the Fermi liquid regime with increasing field. The obtained results therefore lead to the conclusion that the valent degrees of freedom play a minor role in the strong field dependence of the heavy fermion systems in this compound.
\end{abstract}

PACS numbers: 71.27.+a, 75.30.Kz, 75.30.Mb, 78.70.Dm

\section{Introduction}

Many attempts to approach magnetic-nonmagnetic transitions have been made in rare-earth based heavy fermion (HF) materials, for example application of pressure, magnetic field, or chemical doping, because various unconventional properties have been observed in the vicinity of this phase boundary. Among them, recent reports of a magnetic field induced quantum critical point in ytterbium $(\mathrm{Yb})$ based $\mathrm{HF}$ compounds have attracted much interest $[1,2]$. Those studies indicate that, after antiferromagnetic ground state is suppressed by field, Fermi liquid (FL) like behavior appears and is enhanced with increasing field. However, the field effect on $4 f$ electronic states near the magnetic-nonmagnetic transitions is not simple, because it simultaneously affects several degrees of freedom with small characteristic energies, for example interactions between conduction and $f$ electrons, crystalline electric field, valence of rare-earth ions, and so on. Besides, recent theoretical works pointed out that valence fluctuations play an important role in peculiar

\footnotetext{
* corresponding author; e-mail: mito@sci.u-hyogo.ac.jp

† Present address: IAMR, Hiroshima Univ., Higashi Hiroshima 739-8530, Japan.
}

properties in magnetic field observed in the HF materials [3]. However, from experimental point of view, it is not easy to extract the field effect on the valence. For example, in the above mentioned field induced FL states in $\mathrm{YbRh}_{2} \mathrm{Si}_{2}$, with increasing field, the temperature dependence of the susceptibility behaves as if $4 f$ electron system became more itinerant [4], namely analogous to the behavior typically observed in mixed valence states than in a well localized trivalent $\mathrm{Yb}$ state. Actually, the field effect on the valence near the magnetic-nonmagnetic phase boundary has not been studied in detail so far.

In this paper, we report the studies of the X-ray absorption spectroscopy (XAS) measurement, which is known as a powerful tool for determining valence states of ions, on the $\mathrm{HF}$ compound $\mathrm{YbCo}_{2} \mathrm{Zn}_{20}$, and present how the $\mathrm{Yb}$ valence depends upon the field. In contrast to what is observed in cerium (Ce)-based HF compounds, most of the Yb-based HF materials do not show superconductivity in the vicinity of the magneticnonmagnetic phase boundary, which is rather advantageous in terms of investigating the criticality down to very low temperatures. $\mathrm{YbCo}_{2} \mathrm{Zn}_{20}$ crystallizes in the cubic $\mathrm{CeCr}_{2} \mathrm{~A}_{20}$ structure with $\mathrm{Yb}-\mathrm{Yb}$ distance $\approx 6.0 \AA$. One of the most unique features of this compound is an extremely large specific heat at low temperatures $\left(C / T=7.9 \mathrm{~J} /\left(\mathrm{mol} \mathrm{K}^{2}\right)\right)$, whose origin has not been clarified yet, with no sign of long range magnetic order down 
to the lowest temperature [5]. Besides, pressure induced magnetic ordering with a low critical pressure of $\approx 1 \mathrm{GPa}$ is suggested by the resistivity and specific heat measurements $[6,7]$. These facts indicate that this compound locates very close to the magnetic-nonmagnetic border at ambient pressure.

\section{Experimental}

Single crystal samples of $\mathrm{YbCo}_{2} \mathrm{Zn}_{20}$ were grown using the $\mathrm{Zn}$ self-flux method as reported previously [6]. The single crystal sample was finely powdered for the XAS experiments. The measurements were performed in the temperature range of 2 to $300 \mathrm{~K}$ and in the field range of 0 to $10 \mathrm{~T}$ at a beam line BL39XU of SPring-8 in Japan. The XAS spectra at the $\mathrm{Yb} L_{3}$-edge were recorded in the transmission mode using ionization chambers. The energy was calibrated with the XAS spectrum of $\mathrm{Yb}_{2} \mathrm{O}_{3}$ at room temperature.

\section{Results and discussion}

Figure 1 shows the $L_{3}$-edge absorption spectra in zero field measured at 286 and $2 \mathrm{~K}$. The main peak at $8.947 \mathrm{keV}$ corresponds to the trivalent component. The signal for the divalent state, which generally appears around $8.939 \mathrm{keV}$ [8], is not clearly observed in the present spectra. The $\mathrm{Yb}$ valence therefore should be very close to +3 , although the precise absolute value could not be determined because very little spectral weight of the divalent component led to the difficulty in fitting the data. When temperature is lowered from 286 to $2 \mathrm{~K}$, there is no difference in the lineshape within experimental error. This is consistent with the fact that the susceptibility of $\mathrm{YbCo}_{2} \mathrm{Zn}_{20}$ follows the Curie-Weiss law down to $2 \mathrm{~K}$ with an effective moment near the full free-ion moment of $\mathrm{Yb}^{3+}[5,7]$. We also note that, in the temperature dependence of $C / T$, the FL behavior with the heavy mass is seen only below $0.2 \mathrm{~K}[5,7]$.

We have found that the absorption spectrum is insensitive to the application of field. As shown in Fig. 2, the spectrum measured at $2 \mathrm{~K}$ does not reveal any changes in shape when the field is applied up to $10 \mathrm{~T}$, implying that we observed no evidence that the original $\mathrm{Yb}$ trivalent valence changes in the magnetic field. Here the resolution of the absorption spectrum corresponds typically to the valence of 0.01 or less.

This trend obviously contrasts to strong field dependences observed in the resistivity and the susceptibility $(M / H)$. As field increases, the FL regime in the resistivity, where the resistivity $\rho$ follows $\rho=\rho_{0}+A T^{2}$, rapidly extends to higher temperature range, and the $A$ value becomes about $10^{2}$ times smaller at $6 \mathrm{~T}$ [9]. $M / H$ deviates downward from the Curie-Weiss law with increasing field above $1 \mathrm{~T}(M / H$ at $7 \mathrm{~T}$ is $\approx 50 \%$ of that at $1 \mathrm{~T})$, and becomes less temperature dependent at low temperatures (not shown). Such field induced FL behavior is also observed in $\mathrm{YbRh}_{2} \mathrm{Si}_{2}[2,4,10]$. From these experimental facts, we conclude that the significant field effects

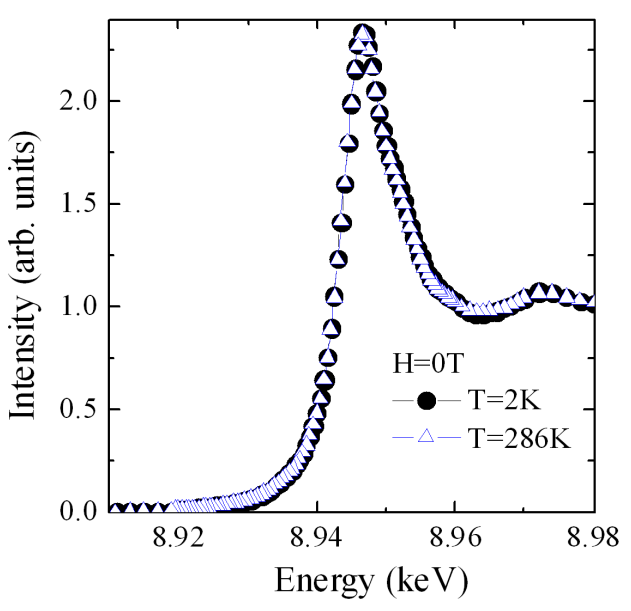

Fig. 1. $L_{3}$-edge absorption spectra of $\mathrm{YbCo}_{2} \mathrm{Zn}_{20}$ powder sample taken at 2 and $286 \mathrm{~K}$ in zero field.

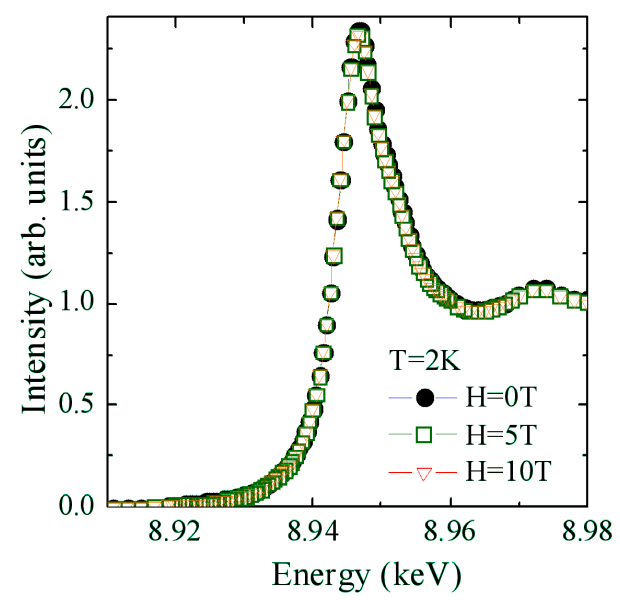

Fig. 2. Field dependence of the $L_{3}$-edge absorption spectrum of $\mathrm{YbCo}_{2} \mathrm{Zn}_{20}$ measured at fields of 0,5 , and $10 \mathrm{~T}$ and at the constant temperature of $2 \mathrm{~K}$.

in $\mathrm{YbCo}_{2} \mathrm{Zn}_{20}$ near the magnetic-nonmagnetic transition result from the interactions not between the field and the valence of $\mathrm{Yb}$ ions, but between the field and magnetic moments of the $4 f$ electrons. The enhanced FL behavior with increasing field is probably attributed to large suppression of spin fluctuations under the field.

\section{Conclusions}

In summary, we have performed the XAS measurements on the $\mathrm{HF}$ compound $\mathrm{YbCo}_{2} \mathrm{Zn}_{20}$ up to $10 \mathrm{~T}$ in order to investigate the possibility that the application of magnetic field gives rise to changes in the $\mathrm{Yb}$ valence in the vicinity of the magnetic-nonmagnetic transition. Although the application of field enhances the FL behavior in $4 f$ electron systems, we obtained no evidence for changes in the $\mathrm{Yb}$ valence within experimental error. Our results indicate that field induced modifications in 
magnetic properties are mainly attributed to large suppression of spin fluctuations.

\section{Acknowledgments}

This work was partially supported by Grant-in-Aid for Scientific Research (grant No. 21540342) and Scientific Research on Innovative Areas "Heavy Electrons" (grant No. 21102522) from the Ministry of Education, Culture, Sports, Science and Technology of Japan. The XAS measurements at BL39XU (proposal No. 2009B-1544) in SPring-8 were performed with the approval of the Japan Synchrotron Radiation Research Institute (JASRI).

\section{References}

[1] E. Bauer, R. Hauser, A. Galatanu, H. Michor, G. Hilscher, J. Sereni, M.G. Berisso, P. Pedrazzini, M. Galli, F. Marabelli, P. Bonville, Phys. Rev. B 60, 1238 (1999).

[2] P. Gegenwart, J. Custers, C. Geibel, K. Neumaier, T. Tayama, K. Tenya, O. Trovarelli, F. Steglich, Phys. Rev. Lett. 89, 056402 (2002).

[3] S. Watanabe, A. Tsuruta, K. Miyake, J. Flouquet, J. Phys. Soc. Jpn. 78, 104706 (2009).
[4] O. Trovarelli, C. Geibel, S. Mederle, C. Langhammer, F.M. Grosche, P. Gegenwart, M. Lang, G. Sparn, F. Steglich, Phys. Rev. Lett. 85, 626 (2000).

[5] M.S. Torikachvili, S. Jia, E.D. Mun, S.T. Hannahs, R.C. Black, W.K. Neils, D. Martien, S.L. Bud'ko, P.C. Canfield, Proc. Natl. Acad. Sci. USA 104, 9960 (2007).

[6] Y. Saiga, K. Matsubayashi, T. Fujiwara, M. Kosaka, S. Katano, M. Hedo, T. Matsumoto, Y. Uwatoko, J. Phys. Soc. Jpn. 77, 053710 (2008).

[7] Y. Saiga, Ph.D. Thesis, Saitama University, 2008.

[8] K. Yamamoto, H. Yamaoka, N. Tsujii, A.M. Vlaicu, H. Oohashi, S. Sakakura, T. Tochio, Y. Ito, A. Chainani, S. Shin, J. Phys. Soc. Jpn. 76, 124705 (2007).

[9] Y. Saiga, K. Matsubayashi, T. Fujiwara, T. Matsumoto, M. Kosaka, S. Katano, Y. Uwatoko, J. Phys., Conf. Ser. 150, 042168 (2009).

[10] K. Ishida, K. Okamoto, Y. Kawasaki, Y. Kitaoka, O. Trovarelli, C. Geibel, F. Steglich, Phys. Rev. Lett. 89, 107202 (2002). 